\title{
MULTIFRACTAL ANALYSIS FOR CONVOLUTIONS OF OVERLAPPING CANTOR MEASURES*
}

\author{
VICTOR POK-WAI FONG ${ }^{\dagger}$, KATHRYN E. HARE ${ }^{\ddagger}$, AND DANIEL L. JOHNSTONE ${ }^{\S}$
}

\begin{abstract}
Unlike the case for self-similar measures satisfying the open set condition, it has been shown that the $m$-fold convolution of the uniform Cantor measure on the classical middlethird Cantor set has isolated points in its multifractal spectrum for any $m \geq 3$. We show that this phenomena of isolated points holds for quite general Cantor measures on Cantor sets that can be far from self-similar.

We also prove, in contrast, that if the convolution is understood on the group $[0,1]$, rather than on $\mathbb{R}$, then the multifractal spectrum of the 3 -fold convolution of the uniform Cantor measure is an interval.
\end{abstract}

Key words. Multifractal analysis, local dimension, Cantor measure, convolution.

AMS subject classifications. Primary 28A80, 28A78; Secondary 42A36.

1. Introduction. A useful tool in the study of singular measures is the concept of the local dimension of the measure $\mu$ defined at points $x$ in the support of $\mu$ by

$$
\operatorname{dim}_{l o c} \mu(x)=\lim _{r \rightarrow 0^{+}} \frac{\log \mu(B(x, r))}{\log r} .
$$

For measures that are suitably uniform the local dimension can be the same value at every point in the support of the measure, but for more general measures it is of interest to determine for which $\alpha$ the sets $E_{\alpha}=\left\{x: \operatorname{dim}_{\text {loc }} \mu(x)=\alpha\right\}$ are non-empty, the so-called multifractal spectrum, and to quantify the size of these sets. This has been done for many examples of measures, including (quasi) self-similar measures and $p$-Cantor measures on central Cantor sets which satisfy a separation condition (the open set condition in the case of self-similar measures). For such measures it is known that the multifractal spectrum is an interval and there is a formula for calculating the dimensions of the sets $E_{\alpha}$, known as the multifractal formalism (c.f., [2], [6], [9], [10]).

The uniform Cantor measure, $\mu$, supported on the classical middle-third Cantor set, and its $m$-fold convolutions, denoted $\mu^{m}$, are interesting examples of self-similar measures generated by the iterated function systems (IFS) $\left\{F_{i}(x)=x / 3+2 i / 3\right\}$ acting on $[0, m]$, with probabilities $\left\{2^{-m}\left(\begin{array}{c}m \\ i\end{array}\right)\right\}$, for $i=0,1, \ldots, m$. The Cantor measure $\mu$ has the same local dimension at all points of its support. When $m=2$, the open set condition is satisfied and the multifractal spectrum of $\mu^{2}$ can be obtained through the multifractal formalism.

However, if $m \geq 3$ the open set condition does not hold and in [7] Hu and Lau discovered the striking fact that the multifractal spectrum of $\mu^{m}$ is not an interval. In fact, they showed that $\operatorname{dim}_{l o c} \mu^{m}(0)$ is an isolated point in the multifractal spectrum and is the maximum local dimension.

\footnotetext{
*Received February 4, 2010; accepted for publication December 9, 2010. This research was supported in part by NSERC and by the Chinese University of Hong Kong. This paper is in final form and no version of it will be submitted for publication elsewhere.

${ }^{\dagger}$ Department of Mathematics, The Chinese University of Hong Kong, Shatin, Hong Kong (victory fong@yahoo.com.hk).

${ }^{\ddagger}$ Department of Pure Mathematics, University of Waterloo, Waterloo, Ont., Canada N2L 3G1 (kehare@uwaterloo.ca).

$\S$ Department of Pure Mathematics, University of Waterloo, Waterloo, Ont., Canada N2L 3G1 (danielljohnstone@gmail.com).
} 
Their work was generalized by Shmerkin in [11], who proved that there is an isolated point in the multifractal spectrum of self-similar measures generated by IFS having overlap and of the form $\{x / d+i / d: i=0, \ldots, m\}$ with integer $d \geq 3$, and probabilities $p_{i}$ satisfying $p_{0}, p_{m} \leq p_{i}$ for all $i$.

We consider more general Cantor sets and Cantor measures which can be far from self-similar. We allow the number of removed intervals and the ratios of dissection to vary at each step in the construction, requiring only that the Cantor intervals of a given step have the same length and are equally spaced apart, and that the ratios of dissection are bounded away from zero. We also allow the probabilities defining the Cantor measure to vary. (The precise definitions are given in Section 2.) Examples include $p$-Cantor measures on central Cantor sets and Cantor measures whose weights are uniformly distributed across the Cantor intervals of each step.

The boundedness of the ratios of dissection ensures that our Cantor sets, $C$, have the property that $(M) C=[0, M]$ for a sufficiently large $M$. In section 3 we show that if $m \geq M+2$, then $\operatorname{dim}_{l o c} \mu^{m}(0)$ is isolated and maximal in the multifractal spectrum of $\mu^{m}$ provided the Cantor interval containing 0 at each step in the construction has minimal $\mu$ measure. (This assumption is analogous to Shmerkin's assumption that $p_{0} \leq p_{i}$.) Our results are not as sharp as [7] or [11], but this is not surprising as our Cantor sets and measures do not, in general, have the same rigid combinatorial properties.

In harmonic analysis it is often of interest to consider convolution on the group $[0,1]$ under addition mod 1 rather than $\mathbb{R}$. In contrast to [7], in section 4 we prove that if $\mu$ is the classical Cantor measure and the convolution is taken on $[0,1]$, then $\mu^{3}$ has no isolated points in its multifractal spectrum and $\operatorname{dim}_{l o c} \mu^{3}(0)$ is the minimum local dimension. In fact, we show that the set of local dimensions is a proper subinterval of the multifractal spectrum of $\mu^{3}$ when the convolution is taken in $\mathbb{R}$. Similar statements can be made for $\mu^{m}$, when $\mu$ is the uniform Cantor measure on the Cantor set with (fixed) ratio of dissection $1 / m, m \geq 4$.

Other related results can be found, for example, in [3], [4], [8] and [12].

\section{Cantor sets and measures.}

2.1. Cantor set construction. Given a sequence of positive integers $k_{j}$ and real numbers $r_{j}, g_{j} \in(0,1)$ such that

$$
\left(k_{j}+1\right) r_{j}+k_{j} g_{j}=1
$$

we construct the uniform Cantor set $C\left(\left\{k_{j}\right\},\left\{r_{j}\right\}\right)$ as follows. We begin with $C_{0}=$ $[0,1]$. Remove from $C_{0}$ the $k_{1}$ equally spaced open intervals of length $g_{1}$, called the gaps of step one. The union of the $k_{1}+1$ remaining closed intervals, each of length $r_{1}$, called the Cantor intervals of step one, will be denoted $C_{1}$. Next, from each step one Cantor interval remove the gaps of step two, the $k_{2}$ equally spaced open intervals of length $g_{2} r_{1}$, leaving a total of $\left(k_{1}+1\right)\left(k_{2}+1\right)$ closed intervals each of length $r_{1} r_{2}$, the step two Cantor intervals. The union of the step two intervals will be denoted $C_{2}$. In general, $C_{j}$ consists of the $\left(k_{1}+1\right) \cdots\left(k_{j}+1\right)$ closed step $j$ Cantor intervals, of length

$$
R_{j} \equiv r_{1} \cdots r_{j}
$$

equally spaced in the step $j-1$ Cantor intervals. The uniform Cantor set $C=$ $C\left(\left\{k_{j}\right\},\left\{r_{j}\right\}\right)=\cap_{j=1}^{\infty} C_{j}$. 
This construction yields a compact, perfect, totally disconnected subset of $[0,1]$, whose Hausdorff and (lower and upper) box dimensions are easily seen to be given by

$$
\begin{aligned}
& \operatorname{dim}_{H} C=\underline{\operatorname{dim}}_{B} C=\liminf _{n} \frac{\log \prod_{j=1}^{n}\left(k_{j}+1\right)}{\left|\log R_{n}\right|} \text { and } \\
& \overline{\operatorname{dim}}_{B} C=\limsup _{n} \frac{\log \prod_{j=1}^{n}\left(k_{j}+1\right)}{\left|\log R_{n}\right|} .
\end{aligned}
$$

We note that every element in $C$ can be presented as $\sum_{j=1}^{\infty} R_{j-1}\left(r_{j}+g_{j}\right) x_{j}$ with $x_{j} \in\left\{0,1, \ldots, k_{j}\right\}$. For example, $1=\sum_{j=1}^{\infty} R_{j-1}\left(r_{j}+g_{j}\right) k_{j}$. We also remark that as $k_{j} \geq 1$ and $g_{j}>0$ we have $r_{j}<1 / 2$.

There is a natural way to label the Cantor intervals of step $n$ by the words $\omega=\left(\omega_{1}, \ldots, \omega_{n}\right)$ of length $n$, with $\omega_{j} \in\left\{0,1, \ldots, k_{j}\right\}$. We denote the intervals of step one as $I_{0}, \ldots, I_{k_{1}}$, listing these from left to right. Given an interval $I_{\omega}$ of step $n$, we label the step $n+1$ Cantor subintervals of $I_{\omega}$ (from left to right) as $I_{\omega, 0}, \ldots, I_{\omega, k_{n+1}}$ where by $\omega, i$ we mean the word of length $n+1$ obtained by adjoining the letter $i$ to the word $\omega$.

2.2. Cantor measures. Suppose $p_{i j}>0$ for $i=0, \ldots, k_{j}$ and $\sum_{i=0}^{k_{j}} p_{i j}=1$. By a Cantor measure, $\mu=\mu\left(C,\left\{p_{i j}\right\}\right)$, we mean the probability measure supported on the uniform Cantor set $C=C\left(\left\{k_{j}\right\},\left\{r_{j}\right\}\right)$, whose distribution function is given by

$$
S=\sum_{j=1}^{\infty} R_{j-1}\left(r_{j}+g_{j}\right) X_{j}
$$

where $X_{j}$ are independent random variables taking on the values $\left\{0, \ldots, k_{j}\right\}$ with probabilities $P\left(X_{j}=i\right)=p_{i j}$ for $i=0, \ldots, k_{j}$. Note that the range of $S$ is the Cantor set and $S=1$ if and only if $X_{j}=k_{j}$ for all $j$.

The labelling by words is also convenient for describing the measure $\mu$. If $\omega=$ $\left(\omega_{1}, \ldots, \omega_{n}\right)$, then $\mu\left(I_{\omega}\right)=\prod_{j=1}^{n} p_{\omega_{j} j}$.

2.3. Examples. If $p_{i j}=1 /\left(k_{j}+1\right)$ for all $i, j$, then we call $\mu\left(C,\left\{p_{i j}\right\}\right)$ the uniform Cantor measure as it assigns equal mass, $\prod_{j=1}^{n}\left(k_{j}+1\right)^{-1}$, to each of the step $n$ Cantor intervals.

By a central Cantor set, $C\left(r_{j}\right)$, we mean a Cantor set $C\left(1,\left\{r_{j}\right\}\right)$, i.e., out of each 'parent' interval of step $j-1$ we remove one centred gap and keep the two outer closed intervals of length $r_{1} \cdots r_{j}$. The numbers $r_{j}$ are often called the ratios of dissection and they uniquely determine the Cantor set. The classical middle-third Cantor set is the central Cantor set with ratios $1 / 3$ at each step.

The probability measure on a central Cantor set with $p_{0 j}=p$ and $p_{1 j}=1-p$ for all $j$ is called a $p$-Cantor measure. Of course, when $p=1 / 2$, then the $p$-Cantor measure is the uniform measure on the central Cantor set.

Yet another special case is the self-similar set and measure given by the probabilities $\left\{p_{i}\right\}_{i=0}^{k}$ and the iterated function system $F_{i}(x)=r x+i(r+g)$ where $i=0, \ldots, k$ and $(k+1) r+k g=1$.

\subsection{Sums of Cantor sets and convolutions of Cantor measures. Let}

$$
S^{(m)}=\sum_{j=1}^{\infty} R_{j-1}\left(r_{j}+g_{j}\right)\left(X_{j}^{(1)}+\cdots+X_{j}^{(m)}\right)=\sum_{j=1}^{\infty} R_{j-1}\left(r_{j}+g_{j}\right) Z_{j}^{(m)}
$$


and

$$
S_{N}^{(m)}=\sum_{j=1}^{N} R_{j-1}\left(r_{j}+g_{j}\right) Z_{j}^{(m)},
$$

where the functions $\left\{X_{j}^{(i)}\right\}_{i, j}$ are independent random variables and with the same distribution as $X_{j}$. Thus $Z_{j}^{(m)}$ takes on the values $\left\{0,1, \ldots, m k_{j}\right\}$ and the range of $S^{(m)}$ is a subset of $[0, m]$ (with equality if $(m) C=[0, m]$ ).

Since $Z_{j}=0$ if and only if each $X_{j}^{(i)}=0$, and $Z_{j}^{(m)}=m k_{j}$ if and only if each $X_{j}^{(i)}=k_{j}, P\left(Z_{j}^{(m)}=0\right)=p_{0 j}^{m}$ and $P\left(Z_{j}^{(m)}=m\right)=p_{k_{j} j}^{m}$. More generally, if we let $p_{j}=\min _{i} p_{i j}$, then it is easy to see that $P\left(Z_{j}^{(m)}=z\right)$ is at least $p_{j}^{m}$ times the number of ways of obtaining $z$ as $\sum_{i=1}^{m} z_{i}$ with $z_{i} \in\left\{0, \ldots, k_{j}\right\}$.

The $m$-fold convolution product of $\mu$, denoted $\mu^{m}$, has $S^{(m)}$ as its distribution function. This measure is supported on $(m) C$, the $m$-fold sum of $C$. The measures, $\mu_{N}^{m}$, whose distribution functions are given by $S_{N}^{(m)}$, converge weakly to $\mu^{m}$.

Every element of $(m) C$ can be written as $z=\sum_{j=1}^{\infty} R_{j-1}\left(r_{j}+g_{j}\right) z_{j}$ with $z_{j} \in$ $\left\{0, \ldots, m k_{j}\right\}$, however the choice of $z_{j}$ need not be unique. Given such a presentation we will let

$$
\sigma_{n}(z)=\sum_{j=1}^{n} R_{j-1}\left(r_{j}+g_{j}\right) z_{j}
$$

It should be clear from the context whether we intend a particular presentation or an arbitrary presentation when we use this notation.

Throughout this paper we will assume

$$
\rho \equiv \inf _{j} r_{j}>0
$$

Of course, this implies $\sup _{j} k_{j}<\infty$. This assumption guarantees that there exists a positive integer $M$ such that $(M) C=[0, M]$ (see [5]). In fact, we can take any $M \geq \sup \left(k_{j} r_{j}\right)^{-1}-1$. (For example, $M=2$ suffices in the case of the classical middlethird Cantor set.) We note that as $0,1 \in C$, if $(M) C=[0, M]$, then $(m) C=[0, m]$ for all $m \geq M$.

3. Local dimensions of convolutions of Cantor measures on $\mathbb{R}$. Given any measure $\nu$ on $\mathbb{R}^{n}$ and $x \in \operatorname{supp} \nu$, we define the lower local dimension of $\nu$ at $x$ by

$$
\underline{\operatorname{dim}}_{l o c} \nu(x)=\liminf _{r \rightarrow 0^{+}} \frac{\log \nu(B(x, r))}{\log r}
$$

and define the upper local dimension, $\overline{\operatorname{dim}}_{l o c} \nu(x)$, and the local dimension, $\operatorname{dim}_{l o c} \nu(x)$, by taking the limsup or limit respectively. Our interest will be in measures defined on $\mathbb{R}$, so $B(x, r)=(x-r, x+r)$.

Lemma 1. Suppose $\mu=\mu\left(C,\left\{p_{i j}\right\}\right)$ is a Cantor measure. For any $z \in(m) C$,

$$
\underline{\operatorname{dim}}_{l o c} \mu^{m}(z) \leq \liminf _{n \rightarrow \infty} \frac{\log \mu_{n}^{m}\left(\sigma_{n}(z)\right)}{\log R_{n}}
$$

where $\sigma_{n}(z)$ can be taken with respect to any presentation of $z$. If we replace lim inf by lim sup we obtain an upper bound on the upper local dimension. 
Proof. As $\left(r_{j}+g_{j}\right) k_{j}=1-r_{j}$, we can deduce from the telescoping sum that

$$
\begin{aligned}
\left|z-\sigma_{n}(z)\right| & \leq \sum_{j=n+1}^{\infty} R_{j-1}\left(r_{j}+g_{j}\right) k_{j} m \\
& =\sum_{j=n+1}^{\infty} R_{j-1}\left(1-r_{j}\right) m=m R_{n} .
\end{aligned}
$$

Thus $\sigma_{n}(z) \in B\left(z, R_{n} m\right)$.

More generally, if $y \in(m) C$ and $\sigma_{n}(y) \in B\left(z, m R_{n}\right)$, then $y \in B\left(z, 2 m R_{n}\right)$. Hence

$$
\mu_{n}^{m}\left(\sigma_{n}(z)\right) \leq \mu_{n}^{m}\left(B\left(z, m R_{n}\right)\right) \leq \mu^{m}\left(B\left(z, 2 m R_{n}\right)\right) .
$$

Given any $r>0$, choose $n$ such that

$$
2 m R_{n} / \rho \geq r \geq 2 m R_{n+1} / \rho \geq 2 m R_{n} .
$$

It follows that $\mu^{m}\left(B\left(z, 2 m R_{n}\right)\right) \leq \mu^{m}(B(z, r))$, thus,

$$
\begin{aligned}
\underline{\operatorname{dim}}_{l o c} \mu^{m}(z) & =\liminf _{r \rightarrow 0^{+}} \frac{\log \mu^{m}(B(z, r))}{\log r} \\
& \leq \liminf _{n \rightarrow \infty} \frac{\log \left(\mu_{n}^{m}\left(\sigma_{n}(z)\right)\right)}{\log 2 m R_{n} / \rho} \\
& =\liminf _{n \rightarrow \infty} \frac{\log \left(\mu_{n}^{m}\left(\sigma_{n}(z)\right)\right.}{\log R_{n}},
\end{aligned}
$$

with the last equality holding because $m$ and $\rho$ are fixed.

The proof for the upper local dimension is similar.

Lemma 2. Suppose $\mu=\mu\left(C,\left\{p_{i j}\right\}\right)$. The lower local dimensions of $\mu^{m}$ at 0 and $m$ are given by

$$
\underline{\operatorname{dim}}_{l o c} \mu^{m}(0)=\liminf _{n} \frac{m \log \prod_{j=1}^{n} p_{0 j}}{\log R_{n}}=m \underline{\operatorname{dim}}_{l o c} \mu(0)
$$

and

$$
\underline{\operatorname{dim}}_{l o c} \mu^{m}(m)=\liminf _{n} \frac{m \log \prod_{j=1}^{n} p_{k_{j} j}}{\log R_{n}}=m \underline{\operatorname{dim}}_{l o c} \mu(1) .
$$

For the upper local dimensions, just replace lim inf by lim sup.

Proof. As $\sigma_{n}(0)=0$ for all $n$, the first lemma implies

$$
\underline{\operatorname{dim}}_{l o c} \mu^{m}(0) \leq \liminf _{n} \frac{\log \mu_{n}^{m}(0)}{\log R_{n}} .
$$

It is easy to see that $\mu_{n}^{m}(0)=\prod_{j=1}^{n} p_{0 j}^{m}$, hence

$$
\underline{\operatorname{dim}}_{l o c} \mu^{m}(0) \leq \liminf _{n} \frac{\log \prod_{j=1}^{n} p_{0 j}^{m}}{\log R_{n}} .
$$

For the reverse inequality, suppose $z \in(m) C \cap B\left(0, R_{n}\right)$. Let $k_{0}=1+\sup _{j} k_{j}$ and choose $K$ such that $2^{-K}<1 / k_{0}$. If $\sigma_{n-K}(z) \neq 0$, then $z \geq \sigma_{n-K}(z) \geq R_{j-1}\left(r_{j}+g_{j}\right)$ 
for some $j \leq n-K$. But $r_{j}+g_{j} \geq 1 /\left(k_{j}+1\right)$, thus $z \geq R_{n-K} / k_{0}$. Since $r_{j} \leq 1 / 2$, the choice of $K$ ensures that $z \leq R_{n} \leq R_{n-K} 2^{-K}<R_{n-K} / k_{0}$. This contradiction shows that we must have $\sigma_{n-K}(z)=0$, hence

$$
\left\{x: S(x) \in B\left(0, R_{n}\right)\right\} \subseteq\left\{x: S_{n-K}(x)=0\right\} .
$$

Therefore

$$
\prod_{j=1}^{n-K} p_{0 j}^{m}=\mu_{n-K}^{m}(0) \geq \mu^{m}\left(B\left(0, R_{n}\right)\right) .
$$

Since $K$ is fixed, $R_{n}$ and $R_{n-K}$ are comparable, thus

$$
\begin{aligned}
\underline{\operatorname{dim}}_{l o c} \mu^{m}(0) & =\liminf _{n} \frac{\log \mu^{m}\left(B\left(0, R_{n}\right)\right)}{\log R_{n}} \\
& \geq \liminf _{n} \frac{\log \prod_{j=1}^{n-K} p_{0 j}^{m}}{\log R_{n}}=\liminf _{n} \frac{m \log \prod_{j=1}^{n} p_{0 j}}{\log R_{n}} .
\end{aligned}
$$

The other cases are similar.

Using formulas (2.1) we immediately obtain the following.

Corollary 1. If $\mu$ is the uniform Cantor measure, then

$$
\underline{\operatorname{dim}}_{l o c} \mu^{m}(0)=\underline{\operatorname{dim}}_{l o c} \mu^{m}(m)=m \operatorname{dim}_{H} C
$$

and

$$
\overline{\operatorname{dim}}_{l o c} \mu^{m}(0)=\overline{\operatorname{dim}}_{l o c} \mu^{m}(m)=m \overline{\operatorname{dim}}_{B} C .
$$

Corollary 2. If $\mu$ is the $p$-Cantor measure supported on a central Cantor set, then

$$
\underline{\operatorname{dim}}_{l o c} \mu^{m}(0)=\liminf _{n} \frac{m n \log p}{\log R_{n}}
$$

and

$$
\underline{\operatorname{dim}}_{l o c} \mu^{m}(m)=\liminf _{n} \frac{m n \log (1-p)}{\log R_{n}} .
$$

From the next result we will deduce the existence of an isolated point in the spectrum. The notation $\lceil z\rceil$ denotes the least integer greater or equal to $z$.

Theorem 1. Assume $C=C\left(\left\{k_{j}\right\},\left\{r_{j}\right\}\right)$ is a uniform Cantor set with $\inf r_{j}>$ 0 and that $\mu=\mu\left(C,\left\{p_{i j}\right\}\right)$ is a Cantor measure. Suppose $M$ is chosen such that $(M) C=[0, M]$. Let $p_{j}=\min _{i} p_{i j}$. For any $m \geq M+2$ and any $x \in \operatorname{supp} \mu^{m}$, $x \neq 0, m$, we have

$$
\underline{\operatorname{dim}}_{l o c} \mu^{m}(x) \leq \liminf _{n} n\left(\frac{m \log \prod_{j=1}^{n} p_{j}^{-1 / n}-\log \left(\underset{\left\lceil\frac{m-M-1}{2}\right\rceil}{m}\right)}{\left|\log R_{n}\right|}\right) .
$$


A similar statement holds for the upper local dimension with lim sup replacing lim inf.

Proof. First, suppose $x \in(0, M]$.

Fix non-negative, bounded integers $b_{j}$ and let $t_{N}=\sum_{j=N}^{\infty} b_{j} R_{j-1}\left(r_{j}+g_{j}\right)$. (Later we will specify $b_{j}$.) Since $t_{N}$ decreases to 0 as $N \rightarrow \infty$, we can choose $N$ such that $0<x-t_{N}=y \leq M$. As $(M) C=[0, M]$, we may represent $y$ as $\sum_{j=1}^{\infty} R_{j-1}\left(r_{j}+g_{j}\right) y_{j}$ with $y_{j} \in\left\{0,1, \ldots, M k_{j}\right\}$. Then

$$
x=t_{N}+y=\sum_{j=1}^{\infty} R_{j-1}\left(r_{j}+g_{j}\right) x_{j}
$$

with $x_{j}=y_{j}+b_{j} \in\left\{b_{j}, b_{j}+1, \ldots, b_{j}+M k_{j}\right\}$ if $j \geq N$ and $x_{j}=y_{j}$ else. Consequently, $x$ has a presentation with its $j$ th digit in the set $\left\{b_{j}, b_{j}+1, \ldots, b_{j}+M k_{j}\right\}$ for all but finitely many $j$.

Now assume $d M<x \leq(d+1) M$ for a positive integer $d$.

Notice that both the Cantor set and its $m$-fold sum are symmetric about the midpoints of the intervals $[0,1]$ and $[0, m]$, respectively. It follows that $\underline{\operatorname{dim}}_{l o c} \mu^{m}(x)=$ $\underline{\operatorname{dim}}_{l o c} \nu^{m}(m-x)$, where $\nu=\nu\left(C,\left\{q_{i j}\right\}\right)$ is the Cantor measure with probabilities $q_{i j}=p_{k_{j}-i, j}$. Thus there is no loss in assuming $x \leq m / 2$.

The arguments now depend on the parity of $m$ and $M$. We will give the details for the case $m$ odd as it illustrates the key ideas.

Case 1: $M$ even.

Subcase (a) $x \leq(d+1 / 2) M$. Put $s=x-(d-1 / 2) M$. The first part of the argument, applied to $s \in(0, M]$, shows that given positive, bounded integers $b_{j}$ we may choose an integer $N$ and digits $s_{j} \in\left\{b_{j}, \ldots, b_{j}+M k_{j}\right\}$ for all $j \geq N$, such that $s=\sum_{j=1}^{\infty} R_{j-1}\left(r_{j}+g_{j}\right) s_{j}$. We do this with the choice

$$
b_{j}=\left(\frac{m-1}{2}-d M\right) k_{j} .
$$

Since $m / 2 \geq x>d M$ and $m$ is odd, $m / 2 \geq d M+1 / 2$, and so $b_{j} \geq 0$, as required.

As $(d-1 / 2) M$ is an integer and $\sum R_{j-1}\left(r_{j}+g_{j}\right) k_{j}=1$, we can write

$$
x=s+(d-1 / 2) M=\sum R_{j-1}\left(r_{j}+g_{j}\right) x_{j}
$$

with $x_{j}=s_{j}+k_{j}(d-1 / 2) M$. Hence for $j \geq N$,

$$
x_{j} \in\left\{\frac{k_{j}(m-M-1)}{2}, \ldots, \frac{k_{j}(m+M-1)}{2}\right\} .
$$

We remark that as $m-M-1$ is even, $x_{j}=P_{j} k_{j}+Q_{j}$, where the integers $P_{j} \in$ $\{(m-M-1) / 2, \ldots,(m+M-1) / 2\}$ and $Q_{j} \in\left\{0,1, \ldots, k_{j}-1\right\}$. Since $m \geq M+2$, we have $P_{j} \geq 1$ and $P_{j}+1 \leq m$.

This observation shows there are at least $\left(\begin{array}{c}m \\ P_{j}\end{array}\right)$ ways of writing $x_{j}$ as $\sum_{l=1}^{m} z_{l}$ with $z_{l} \in\left\{0,1, \ldots, k_{j}\right\}$ (taking $P_{j}$ indices equal to $k_{j}$ and one index to be $Q_{j}$ ). Taking $Z_{j}^{(m)}$ as in section 2.4, it follows that

$$
P\left(Z_{j}^{(m)}=x_{j}\right) \geq p_{j}^{m}\left(\begin{array}{c}
m \\
\frac{m-M-1}{2}
\end{array}\right) \text { for all } j \geq N .
$$


Consequently,

$$
\begin{aligned}
\mu_{n}^{m}\left(\sigma_{n}(x)\right) & =\prod_{j=1}^{n} P\left(Z_{j}^{(m)}=x_{j}\right) \\
& \geq C_{N} \prod_{j=N}^{n} p_{j}^{m}\left(\begin{array}{c}
m \\
\frac{m-M-1}{2}
\end{array}\right)^{n-N}
\end{aligned}
$$

for $C_{N}=\prod_{j=1}^{N-1} p_{j}^{m}>0$. As $N$ and $C_{N}$ are fixed, together with Lemma 1 this proves

$$
\begin{aligned}
\underline{\operatorname{dim}}_{l o c} \mu^{m}(x) & \leq \liminf _{n} \frac{\log \left(\mu_{n}^{m}\left(\sigma_{n}(x)\right)\right)}{\log R_{n}} \\
& \leq \liminf _{n} n\left(\frac{m \log \prod_{j=1}^{n} p_{j}^{-1 / n}-\log (\underset{m-M-1}{m})}{\left|\log R_{N}\right|}\right) .
\end{aligned}
$$

Subcase (b) $x>(d+1 / 2) M$. By similar reasoning there exists an integer $N$ so that we can represent $s=x-d M \in(0, M]$ as $\sum R_{j-1}\left(r_{j}+g_{j}\right) s_{j}$ with $s_{j} \in$ $\left\{b_{j}, \ldots, b_{j}+M k_{j}\right\}$ for all $j \geq N$ and

$$
b_{j}=k_{j}\left(\frac{m-1}{2}-\left(d+\frac{1}{2}\right) M\right) .
$$

Thus again we see $x$ has a presentation with digits in the range $\left\{k_{j}(m-M-\right.$ $\left.1) / 2, \ldots, k_{j}(m+M-1) / 2\right\}$ for all indices $j \geq N$. The calculation for $\underline{\operatorname{dim}}_{l o c} \mu^{m}(x)$ is the same.

Case 2 : $M$ odd. (Then $M \geq 3$.)

Subcase (a) $x \leq(d+1 / 2) M$. We need to only slightly modify the approach of case 1 (a), taking $s=x-(d-1 / 2) M-1 / 2$. Then $s \in(0, M]$ and $x-s \in \mathbb{N}$. We use $b_{j}=k_{j}(m / 2-d M-1 / 2)$ to see that $x$ has a presentation with its $j$ th digit in the set $\left\{k_{j}(m-M) / 2, \ldots, k_{j}(m+M) / 2\right\}$ for all but finitely many $j$.

Subcase (b) $x>(d+1 / 2) M$. Argue in a similar fashion with $s=x-d M$ and $b_{j}=k_{j}(m / 2-(d+1 / 2) M)$.

In the following corollaries we assume the hypotheses of the theorem hold. Similar statements can be made for the upper local dimensions.

Corollary 3. Suppose $p_{0 j}=\min _{i} p_{i j}$ for each $j$, (or $p_{k_{j} j}=\min _{i} p_{i j}$ for each $j)$ then $\underline{\operatorname{dim}}_{l o c} \mu^{m}(0)$ (respectively, $\underline{\operatorname{dim}}_{l o c} \mu^{m}(m)$ ) is isolated in the set of lower local dimensions and is the maximum lower local dimension.

Proof. Assume $p_{0 j}=\min _{i} p_{i j}$ for all $j$. Since $m \geq M+2,\left(\underset{\left\lceil\frac{m-M-1}{2}\right\rceil}{m}\right) \geq m$. Moreover, $R_{n} \geq \rho^{n}$, thus our calculation of $\underline{\operatorname{dim}}_{l o c} \mu^{m}(0)$ in Lemma 2 and the previous theorem imply that for all $x \neq 0, m$,

$$
\underline{\operatorname{dim}}_{l o c} \mu^{m}(x) \leq \underline{\operatorname{dim}}_{l o c} \mu^{m}(0)-m /|\log \rho| .
$$

Corollary 4. If $\mu$ is the uniform Cantor measure on $C$, then $\underline{\operatorname{dim}}_{l o c} \mu^{m}(0)=$ $\underline{\operatorname{dim}}_{l o c} \mu^{m}(m)$ is isolated in the set of lower local dimensions and is the maximum lower local dimension. 
Proof. As $\mu$ is uniform, $p_{i j}=p_{j}$ for all $j$.

Corollary 5. Suppose $\mu$ is the uniform Cantor measure on the central Cantor set $C\left(r_{j}\right)$ and assume $\inf r_{j} \geq 1 / d$ for some positive integer $d$. Then for any $m \geq d+1$ and $x \in \operatorname{supp} \mu^{m}$, we have

$$
\sup _{x \neq 0, m} \underline{\operatorname{dim}}_{l o c} \mu^{m}(x)<m \operatorname{dim}_{H} C=\underline{\operatorname{dim}}_{l o c} \mu^{m}(0)
$$

Proof. For such a Cantor set we have $(M) C=[0, M]$ if $M \geq d-1$ [1]. It follows from the theorem and Cor. 1 that for any $m \geq d+1$ and $x \in \operatorname{supp} \mu^{m}, x \neq 0, m$,

$$
\underline{\operatorname{dim}}_{l o c} \mu^{m}(x) \leq m \operatorname{dim}_{H} C-\frac{\log \left(\left\lceil\frac{m-d)}{2}\right\rceil\right)}{\limsup \frac{1}{n}\left|\log r_{1} \cdots r_{n}\right|}
$$

As we require $m \geq 4$ for the classical middle-third Cantor set/measure, our results in this special case are not as sharp as [7].

Also, we are not able to determine the sharp upper bound on the set of local dimensions other than at $0, m$, however, we can give a theoretical argument to show that for a fixed Cantor measure the gap between the upper local dimension at 0 or $m$ and the others, is monotomic in $m$. First, a general technical fact.

Lemma 3. Suppose $\nu_{j}$ are measures on $\mathbb{R}$. Let $\mu=\nu_{1} * \cdots * \nu_{m}$ and $x_{j} \in \operatorname{supp} \nu_{j}$ be such that $\sum_{j=1}^{m} x_{j}=x \in \operatorname{supp} \mu$. Then $\overline{\operatorname{dim}}_{l o c} \mu(x) \leq \sum_{j=1}^{m} \overline{\operatorname{dim}}_{l o c} \nu_{j}\left(x_{j}\right)$.

Proof. For all $r>0$ and $t_{j} \in \mathbb{R}$ we clearly have $\chi_{B(x, r)}\left(\sum_{j=1}^{m} t_{j}\right) \geq$ $\prod_{j=1}^{m} \chi_{B\left(x_{j}, r / m\right)}\left(t_{j}\right)$, where $\chi_{B}$ denotes the characteristic function of set $B$. Integrating both sides gives $\mu(B(x, r)) \geq \prod \nu_{j}\left(B\left(x_{j}, r / m\right)\right)$. Thus

$$
\frac{\log \mu(B(x, r))}{\log r} \leq \sum_{j=1}^{m} \frac{\log \nu_{j}\left(B\left(x_{j}, r / m\right)\right)}{\log r}
$$

and taking lim sup gives the desired result.

Now suppose $\mu=\mu\left(C,\left\{p_{i j}\right\}\right)$ is a Cantor measure. Put

$$
\lambda_{m}=\sup _{x \neq 0, m} \overline{\operatorname{dim}}_{l o c} \mu^{m}(x) \text { and } \beta_{m}=\max \left(\overline{\operatorname{dim}}_{l o c} \mu^{m}(0), \overline{\operatorname{dim}}_{l o c} \mu^{m}(m)\right) .
$$

Lemma 2 implies that $\beta_{m}=m \beta_{1}$.

LEMma 4. If $(m) C=[0, m]$, then $\lambda_{m+1} \leq \lambda_{m}+\beta_{1}$.

Proof. Let $x \in \operatorname{supp} \mu^{m+1}, x \neq 0, m+1$. If $0 \leq x \leq(m+1) / 2$, then as $x \leq m$ and $\operatorname{supp} \mu^{m}=[0, m]$, we can apply the lemma with $x_{1}=x$ and $x_{2}=0$. It follows that

$$
\overline{\operatorname{dim}}_{l o c} \mu^{m+1}(x) \leq \overline{\operatorname{dim}}_{l o c} \mu^{m}(x)+\overline{\operatorname{dim}}_{l o c} \mu(0) \leq \overline{\operatorname{dim}}_{l o c} \mu^{m}(x)+\beta_{1} .
$$

If, instead, $m+1 \geq x \geq(m+1) / 2$, then $x-1 \in \operatorname{supp} \mu^{m}$, so we apply the lemma with $x_{1}=x-1$ and $x_{2}=1$ to obtain the same conclusion.

Corollary 6. $\beta_{m}-\lambda_{m}$ is increasing in $m$, provided $m \geq M$ where $(M) C=$ $[0, M]$.

Proof. From the previous comments it follows that $\beta_{m+1}-\lambda_{m+1} \geq(m+1) \beta_{1}-$ $\left(\lambda_{m}+\beta_{1}\right)=\beta_{m}-\lambda_{m}$. 
4. Convolutions of Cantor measures on the torus. It is often of interest to study measures defined on the torus, which we can view as $[0,1)$ under addition $\bmod 1$, or equivalently, as the quotient space $\mathbb{R} / \mathbb{Z}$. Given a measure $\mu$ on $\mathbb{R}$, we will let $\tilde{\mu}$ denote the quotient measure,

$$
\tilde{\mu}(E)=\mu\{t:[t] \in E\}
$$

where $[t]$ denotes the equivalence class of $t \in \mathbb{R}$.

In this section we will be primarily interested in the 3 -fold convolution of the uniform Cantor measure on the middle-third Cantor set, $C(1 / 3)$, which we will denote by $\nu$ (rather than $\mu^{3}$, as in the previous section, to simplify notation) and its quotient measure, $\tilde{\nu}$. Since the support of $\nu$ is $[0,3]$ there is no loss in redefining $[t]=\{t+j \in$ $[0,3]: j \in \mathbb{Z}\}$.

We begin with notation. Let $\Omega \equiv\{0,1,2,3\}$ and $\Omega_{0} \equiv\{0,1,2\}$. Given $x=$ $\left(x_{1}, x_{2}, \ldots\right)$ with $x_{j} \in \Omega$ we let

$$
\pi(x) \equiv \sum_{j=1}^{\infty} 3^{-j} 2 x_{j} \text { and } \pi\left(x_{1}, \ldots, x_{n}\right) \equiv \sum_{j=1}^{n} 3^{-j} 2 x_{j} .
$$

Of course, $\pi(x) \in[0,3]$ and conversely every element in $[0,3]$ can be written as $\sum_{j=1}^{\infty} 3^{-j} 2 x_{j}$ where $x_{j} \in \Omega$, although the choice of digits $x_{j}$ need not be unique. Similarly, every $x \in[0,2]$ can be written as $\sum_{j=1}^{\infty} 3^{-j} 2 x_{j}$ where $x_{j} \in \Omega_{0}$. When the meaning is clear we may omit the $\pi$. We will use the notation $(z)_{n}$ for the $n$-tuple $(z, z, \ldots, z) \in \Omega^{n}$.

The measure $\nu$ is the distribution measure of $S=\sum_{j=1}^{\infty} 3^{-j} 2 Z_{j}$ where $\left\{Z_{j}\right\}_{j=1}^{\infty}$ are independent and identically distributed random variables, with

$$
P\left(Z_{j}=i\right)=\frac{1}{8}\left(\begin{array}{l}
3 \\
i
\end{array}\right) \text { for } i \in \Omega .
$$

As in the previous section, we let $\nu_{n}$ be the distribution measure of $S_{n}=\sum_{j=1}^{n} 3^{-j} 2 Z_{j}$.

In [7], $\mathrm{Hu}$ and Lau determined the the multifractal spectrum of $\nu$, the set of real numbers $\alpha$ such that there is some $x \in \operatorname{supp} \nu \operatorname{such}$ that $\operatorname{dim}_{l o c} \nu(x)=\alpha$.

TheOREM 2. [7, Thm. 1.2] Let $\nu$ be the 3-fold convolution of the uniform Cantor measure on $C(1 / 3)$. The multifractal spectrum of $\nu$ is the set

$$
\left[\frac{\log 8 / 3}{\log 3}, \frac{\log 8 / \sqrt{b}}{\log 3}\right] \cup\left\{\frac{\log 8}{\log 3}\right\}
$$

where $b=(7+\sqrt{13}) / 2$. Furthermore, $\log 8 / \log 3$ is the local dimension at (only) 0 and 3 .

In contrast, we will prove that the multifractal spectrum of $\tilde{\nu}$ is a proper subinterval of the interval above and $\operatorname{dim}_{l o c} \tilde{\nu}([0])$ is the minimum of this interval.

THEOREM 3. Let $\nu$ be the 3-fold convolution of the uniform Cantor measure on $C(1 / 3)$ and let $\tilde{\nu}$ be its quotient measure.

(a) Then $\operatorname{dim}_{l o c} \tilde{\nu}([0])=\frac{\log 8 / 3}{\log 3}$.

(b) For all $s \in[0,3]$,

$$
\frac{\log 8 / 3}{\log 3} \leq \underline{\operatorname{dim}}_{l o c} \tilde{\nu}([s]) \leq \overline{\operatorname{dim}}_{l o c} \tilde{\nu}([s]) \leq \frac{\log 8 / \sqrt{6}}{\log 3}
$$


(c) The multifractal spectrum of $\tilde{\nu}$ is the interval

$$
\left[\frac{\log 8 / 3}{\log 3}, \frac{\log 8 / \sqrt{6}}{\log 3}\right] \text {. }
$$

The proofs of the three parts of this theorem involve delicate calculations and occupy most of the remainder of the paper. We begin with elementary relationships between the local dimensions of $\nu$ and $\tilde{\nu}$.

Lemma 5. Let $s \in[0,3]$. Then

$$
\begin{aligned}
& \overline{\operatorname{dim}}_{l o c} \tilde{\nu}([s]) \leq \min \left\{\overline{\operatorname{dim}}_{l o c} \nu(t): t \in[s]\right\} \text { and } \\
& \underline{\operatorname{dim}}_{l o c} \tilde{\nu}([s])=\min \left\{\underline{\operatorname{dim}}_{l o c} \nu(t): t \in[s]\right\} .
\end{aligned}
$$

Furthermore, if $\operatorname{dim}_{l o c} \nu(t)$ exists for all $t \in[s]$, then

$$
\operatorname{dim}_{l o c} \tilde{\nu}([s])=\min \left\{\operatorname{dim}_{l o c} \nu(t): t \in[s]\right\} .
$$

Proof. We note that since the balls, $B(s+j, r)$ for $j \in \mathbb{Z}$, are disjoint for $r<1 / 2$, $\tilde{\nu}\left(B([s], r)=\sum_{t \in[s]} \nu(B(t, r))\right.$. Thus

$$
\varlimsup_{\operatorname{dim}}^{l o c} \tilde{\nu}([s])=\limsup _{r \rightarrow 0^{+}} \frac{\left|\log \sum_{t \in[s]} \nu(B(t, r))\right|}{|\log r|}
$$

and similarly for the lower local dimension. However, for small $r$,

$$
\min _{t \in[s]}|\log 4 \nu(B(t, r))| \leq \mid \log \sum_{t \in[s]} \nu\left(B(t, r)\left|\leq \min _{t \in[s]}\right| \log \nu(B(t, r)) \mid,\right.
$$

and the limiting behaviours of $|\log 4 \nu(B(t, r))| /|\log r|$ and $|\log \nu(B(t, r))| /|\log r|$ coincide.

In [7] it was shown that

$$
\operatorname{dim}_{l o c} \nu(x)=\lim _{n \rightarrow \infty} \frac{\left|\log \nu_{n}\left(\sigma_{n}(x)\right)\right|}{n \log 3}
$$

with analogous statements holding for the upper and lower local dimensions. Similar arguments give

Lemma 6. For $s \in[0,3]$,

$$
\operatorname{dim}_{l o c} \tilde{\nu}([s])=\lim _{n \rightarrow \infty} \frac{\left|\log \sum_{t \in[s]} \nu_{n}\left(\sigma_{n}(t)\right)\right|}{n \log 3}
$$

and similar statements hold for the upper and lower local dimensions.

Terminology. We will say that $\tau \in \Omega^{n}$ is a barrier if for all positive integers $q$ and for all $\sigma \in \Omega^{q}, \nu_{n+q}(\pi(\sigma, \tau))=\nu_{q}(\pi(\sigma)) \nu_{n}(\pi(\tau))$.

This means that if $\pi\left(\sigma_{1}, \sigma_{2}\right)=\pi(\sigma, \tau)$ for $\sigma_{1} \in \Omega^{q}$ and $\sigma_{2} \in \Omega^{n}$, then $\pi\left(\sigma_{1}\right)=\pi(\sigma)$ and $\pi\left(\sigma_{2}\right)=\pi(\tau)$. The reader can easily check that the set of barriers in $\Omega^{n}$ is

$$
\left\{\tau \in \Omega^{n}: \pi\left((1)_{n}\right) \leq \pi(\tau) \leq \pi\left((2)_{n}\right)\right\} .
$$


The notion of a barrier is very useful for us as it facilitates computations.

Proof of Theorem 3(a). Since $1=\pi(1,1, \ldots)$ and $(1) \in \Omega$ is a barrier, $\nu_{n}\left(\sigma_{n}(1)\right)=$ $\left(\nu_{1}(\pi(1))\right)^{n}=(3 / 8)^{n}$. Hence

$$
\operatorname{dim}_{l o c} \nu(1)=\lim _{n \rightarrow \infty} \frac{\left|\log \nu_{n}\left(\sigma_{n}(1)\right)\right|}{n \log 3}=\log (8 / 3) / \log 3 .
$$

By symmetry the same is true for $\operatorname{dim}_{l o c} \nu(2)$. Since it is known that

$$
\operatorname{dim}_{l o c} \nu(0)=\log 8 / \log 3=\operatorname{dim}_{l o c} \nu(3),
$$

Lemma 5 implies $\operatorname{dim}_{l o c} \tilde{\nu}([0])=\operatorname{dim}_{l o c} \nu(1)$.

Terminology. Given $z \in \Omega^{n}$ with $\pi(z) \geq 3^{-n}$, we denote by $z^{*} \in \Omega^{n}$ the $n$-tuple with $\pi\left(z^{*}\right)=\pi(z)-3^{-n} 2$.

Thus $z$ and $z^{*}$ are 'consecutive' members of $\Omega^{n}$ in the natural sense. If $z=$ $\left(z_{1}, \ldots, z_{n}\right)$ and $z_{n} \neq 0$, then $z^{*}=\left(z_{1}, \ldots, z_{n}-1\right)$, but otherwise one needs to 'borrow' in order to do the subtraction. It will be helpful to obtain good comparisons of $\nu_{n}(\pi(z))$ and $\nu_{n}\left(\pi\left(z^{*}\right)\right)$.

LEMMA 7. There is a decreasing sequence, $\left(\delta_{n}\right)_{n=2}^{\infty}$, with limit 0 and bounded by 1 , such that if $z=\left(z_{1}, \ldots, z_{n}\right) \in \Omega_{0}^{n}$ and $z_{1} \geq 1$, then

$$
\frac{1}{2+\delta_{n}} \leq \frac{\nu_{n}\left(\pi\left(z^{*}\right)\right)}{\nu_{n}(\pi(z))} \leq 2+\delta_{n} .
$$

Proof. A listing of all $z \in \Omega_{0}^{2}$ shows that we may take $\delta_{2}=1 / 4$. We proceed by induction and assume the result holds for $n$.

Let $z=\left(z_{1}, \ldots, z_{n+1}\right) \in \Omega_{0}^{n+1}, z_{1} \geq 1$. There are three cases to consider depending upon the three possible values for $z_{n+1}$.

Case (i) $z_{n+1}=2$. Under this assumption $z^{*}=\left(z_{1}, \ldots, z_{n}, 1\right)$. As both (1) and $(2) \in \Omega$ are barriers and $\nu_{1}(\pi(1))=\nu_{1}(\pi(2))$, we obtain

$$
\begin{aligned}
\nu_{n+1}(z) & =\nu_{n}\left(z_{1}, \ldots, z_{n}\right) \nu_{1}(\pi(2)) \\
& =\nu_{n}\left(z_{1}, \ldots, z_{n}\right) \nu_{1}(\pi(1))=\nu_{n+1}\left(z^{*}\right) .
\end{aligned}
$$

Case (ii) $z_{n+1}=0$. The constraint $z_{1} \geq 1$ implies that $\pi(z) \geq 2 / 3$.

If $\pi(z)=2 / 3$, then $z=(1,0, \ldots, 0)$. However, there are $n$ other elements $w \in \Omega^{n+1}$ such that $\pi(w)=\pi(z)$, namely, $(0,3,0, \ldots, 0)$, $(0,2,3,0, \ldots, 0), \ldots,(0,2,2, \ldots, 2,3)$, thus a simple calculation shows

$$
\nu_{n+1}(z)=\frac{3+\frac{3^{n}-1}{2}}{8^{n+1}} .
$$

On the other hand, $w=(0,2, \ldots, 2)$ is the only member of $\Omega^{n+1}$ with the property that $\pi(w)=\pi\left(z^{*}\right)$. As $(2)$ is a barrier, $\nu_{n+1}\left(z^{*}\right)=\nu_{1}(\pi(0))\left(\nu_{1}(\pi(2))\right)^{n}=3^{n} / 8^{n+1}$. Hence in this case there is an even stronger bound,

$$
1 \leq \frac{\nu_{n+1}\left(z^{*}\right)}{\nu_{n+1}(z)} \leq 2 .
$$


If, instead, $\pi(z)>2 / 3$, then $\pi(z)=\pi(w)$ where $w=\left(w_{1}, \ldots, w_{n}, 3\right)$ with $\left(w_{1}, \ldots, w_{n}\right) \in \Omega_{0}^{n}$ and $w_{1} \geq 1$. Thus $z^{*}=\left(w_{1}, \ldots, w_{n}, 2\right)$ and

$$
\nu_{n+1}\left(z^{*}\right)=\nu_{n}\left(w_{1}, \ldots, w_{n}\right) \nu_{1}(\pi(2)) .
$$

Since $\pi\left(z_{1}, \ldots, z_{n}, 0\right)=\pi\left(w_{1}, \ldots, w_{n}, 3\right)$, we obtain

$$
\nu_{n+1}(z)=\nu_{n}\left(w_{1}, \ldots, w_{n}\right) \nu_{1}(\pi(3))+\nu_{n}\left(z_{1}, \ldots, z_{n}\right) \nu_{1}(\pi(0)) .
$$

Notice that $\pi\left(z_{1}, \ldots, z_{n}\right)-3^{-n} 2=\pi\left(w_{1}, \ldots, w_{n}\right)$, meaning $\left(z_{1}, \ldots, z_{n}\right)^{*}=$ $\left(w_{1}, \ldots, w_{n}\right)$, hence the induction assumption implies

$$
\nu_{n}\left(z_{1}, \ldots, z_{n}\right) \leq\left(2+\delta_{n}\right) \nu_{n}\left(w_{1}, \ldots, w_{n}\right) .
$$

Combining these observations with the fact that $\nu_{1}(\pi(3))=\nu_{1}(\pi(0))=\nu_{1}(\pi(2)) / 3$ yields the bound

$$
\begin{aligned}
\nu_{n+1}(z) & \leq \nu_{n}\left(w_{1}, \ldots, w_{n}\right) \frac{\nu_{1}(\pi(2))}{3}\left(3+\delta_{n}\right) \\
& =\nu_{n+1}\left(z^{*}\right)\left(1+\frac{\delta_{n}}{3}\right) .
\end{aligned}
$$

Similarly,

$$
\nu_{n+1}(z) \geq \nu_{n+1}\left(z^{*}\right)\left(\frac{1}{2+\delta_{n+1}}\right)
$$

with $\delta_{n+1}=\delta_{n} /\left(3+\delta_{n}\right)$.

Case (iii) $z_{n+1}=1$. This is similar, but easier.

Corollary 7. (a) If $z=\left(z_{1}, \ldots, z_{n}, 2\right) \in \Omega_{0}^{n+1}$ and $z_{1} \geq 1$, then $\nu_{n+1}(z)=$ $\nu_{n+1}\left(z^{*}\right)$.

(b) There is a decreasing sequence $\lambda_{n} \rightarrow 0$, such that if $z=\left(z_{1}, \ldots, z_{n}, 0\right) \in \Omega_{0}^{n+1}$ and $z_{1} \geq 1$, then $\nu_{n+1}\left(z^{*}\right) \geq\left(1-\lambda_{n}\right) \nu_{n+1}(z)$.

Proof. These correspond to cases (i) and (ii) in the previous proof, with $1-\lambda_{n}=$ $\left(1+\delta_{n} / 3\right)^{-1}$. $\mathrm{c}$

We will use this to prove the key technical result.

LEMMA 8. Assume $s \in(0,2)$, say $s=\pi\left(x_{1}, x_{2}, \ldots\right)$ with $x_{i} \in \Omega_{0}$ for all $i$ and suppose $0<t=2-s=\pi\left(y_{1}, y_{2}, \ldots\right)<2$ with $y_{i}=2-x_{i} \in \Omega_{0}$ for all $i$. Then for each $\varepsilon>0$ there exists a positive constant $c$ such that for all $n$,

$$
\nu_{n}\left(\pi\left(x_{1}, \ldots, x_{n}\right)\right) \nu_{n}\left(\pi\left(y_{1}, \ldots, y_{n}\right)\right) \geq c\left(\frac{6}{64}-\varepsilon\right)^{n} .
$$

Proof. Fix $\varepsilon>0$ and let $\left\{\lambda_{n}\right\}$ be the sequence of the previous corollary. Choose $N_{0} \geq 2$ such that $\lambda_{N_{0}}<\varepsilon$ and so that $s, t \geq 3^{-N_{0}} 2$. The latter property ensures that for some indices $i, j \leq N_{0}, x_{i} y_{j} \neq 0$. Put $N=2 N_{0}+2$.

Let $s_{n}=\left(x_{1}, \ldots, x_{n}\right)$ and $t_{n}=\left(y_{1}, \ldots, y_{n}\right)$. As $\nu_{n}\left(s_{n}\right), \nu_{n}\left(t_{n}\right)>0$, we can pick $c>0$ such that

$$
\nu_{n}\left(s_{n}\right) \nu_{n}\left(t_{n}\right) \geq c\left(\frac{6}{64}-\varepsilon\right)^{n} \text { for } n \leq N
$$


We proceed by induction, assuming the result for all $1 \leq m \leq N+n$ and establishing it for $m+1=N+n+1$. For notational convenience we will put $p_{m}=\nu_{m}\left(s_{m}\right)$ and $q_{m}=\nu_{m}\left(t_{m}\right)$.

There are various cases to consider, depending on the last several digits of $s_{m+1}, t_{m+1}$.

Case 1: $s_{m+1}=\left(s_{m}, 1\right)$ and $t_{m+1}=\left(t_{m}, 1\right)$. This case is easy as $(1) \in \Omega$ is a barrier digit. Hence $p_{m+1}=p_{m} \nu_{1}(\pi(1))$ and similarly for $q_{m+1}$. As $\left(\nu_{1}(\pi(1))\right)^{2}=$ $9 / 64$, the result follows immediately from the induction assumption.

Otherwise, as the digits add to 2, we can assume without loss of generality that $s_{m+1}=2$ and $t_{m+1}=0$. This situation requires more refined analysis.

Case 2a: $s_{m+1}=\left(s_{m-1}, 1,2\right)$ and $t_{m+1}=\left(t_{m-1}, 1,0\right)$. As $(1,2)$ is a barrier, we have $p_{m+1}=p_{m-1} \nu_{2}(1,2)=p_{m-1} 9 / 64$, and because $\pi(1,0)=\pi(0,3)$ we also have $q_{m+1} \geq q_{m-1} \nu_{2}(1,0)=q_{m-1} 4 / 64$. Thus

$$
p_{m+1} q_{m+1} \geq p_{m-1} q_{m-1}\left(\frac{6}{64}\right)^{2} .
$$

Remaining cases: If the second last digits are not 1 , we cannot obtain sharp enough estimates by only considering the last two digits. It will be enough, however, to analyze the last three digits. A key idea is that if for some $k$ we have $x_{i}=0$ for $i=1, \ldots, k$, i.e., $s_{J}=\left((0)_{k}, x_{k+1}, \ldots, x_{J}\right)$, then

$$
\nu_{J}\left(x_{1}, \ldots, x_{J}\right)=\nu_{k}\left((0)_{k}\right) \nu_{J-k}\left(x_{k+1}, \ldots, x_{J}\right) .
$$

This is due to the fact that $\pi\left(s_{J}\right)<3^{-k} 2$ and therefore if also $\pi\left(s_{J}\right)=\pi\left(z_{1}, \ldots, z_{J}\right)$, then $z_{i}=0$ for all $i=1, \ldots, k$.

If, in addition, the last digit of $s_{J}$ is 0 , then Corollary 7(b) implies

$$
\frac{\nu_{J}\left(s_{J}^{*}\right)}{\nu_{J}\left(s_{J}\right)}=\frac{\nu_{J-k}\left(\left(x_{k+1}, \ldots, x_{J}\right)^{*}\right)}{\nu_{J-k}\left(x_{k+1}, \ldots, x_{J}\right)} \geq 1-\lambda_{J-k} .
$$

The definition of $N_{0}$ ensures that if $x_{1}=\cdots=x_{k}=0$, then $k<N_{0}$. Hence if $J \geq 2 N_{0}$ then

$$
\frac{\nu_{J}\left(s_{J}^{*}\right)}{\nu_{J}\left(s_{J}\right)} \geq 1-\lambda_{J-k} \geq 1-\lambda_{N_{0}} \geq 1-\varepsilon
$$

We will show how to use this idea in one of the remaining cases to illustrate the technique.

Case 2b: $s_{m+1}=\left(s_{m-2}, 0,0,2\right), t_{m+1}=\left(t_{m-2}, 2,2,0\right)$. As $s_{m-1}$ ends with digit 0 and $m-1 \geq 2 N_{0},(4.2)$ shows that $\nu_{m-1}\left(s_{m-1}^{*}\right) \geq(1-\varepsilon) p_{m-1}$. Since

$$
p_{m+1}=p_{m-1} \nu_{2}(0,2)+\nu_{m-1}\left(s_{m-1}^{*}\right) \nu_{2}(3,2)
$$

and $\nu_{2}(0,2)=\nu_{2}(3,2)=3 / 64$, we obtain the estimate

$$
p_{m+1} \geq p_{m-1}\left(\frac{3}{64}+(1-\varepsilon) \frac{3}{64}\right) \geq p_{m-1}\left(\frac{6}{64}-\varepsilon\right) \text {. }
$$

But $(2,0)$ is a barrier and $\pi(2,0)=\pi(1,3)$, thus we can also calculate that $q_{m+1}=$ $q_{m-1} \nu_{2}(2,0)=q_{m-1} 6 / 64$. Consequently,

$$
p_{m+1} q_{m+1} \geq p_{m-1} q_{m-1}\left(\frac{6}{64}-\varepsilon\right) \frac{6}{64}
$$


and the induction assumption gives the desired result.

The other cases are similar.

Proof of Theorem 3(b). As noted in Theorem 2, Hu and Lau show that $\underline{\operatorname{dim}}_{l o c} \nu(s) \geq \log (8 / 3) / \log 3$ for all $s \in[0,3]$, hence the lower bound follows directly from Lemma 5 .

We already know $\operatorname{dim}_{l o c} \tilde{\nu}(0)=\log (8 / 3) / \log 3$, so in verifying the upper bound symmetry allows us to assume that $s \in(0,2)$. Thus $s=\pi\left(x_{1}, x_{2}, \ldots\right)$ where $x_{j} \in \Omega_{0}$. Put $t=2-s=\pi\left(y_{1}, y_{2}, \ldots\right)$ with $y_{i}=2-x_{i}$, so that also $y_{i} \in \Omega_{0}$ for all $i$. We are in the situation of the previous lemma.

Let $u=3-t=1+s$, say $u=\pi\left(z_{1}, z_{2}, \ldots\right)$ with $z_{i}=3-y_{i}$. Of course, $u$ and $s$ belong to the same equivalence class and by symmetry $\nu_{n}\left(u_{n}\right)=\nu_{n}\left(z_{1}, \ldots, z_{n}\right)=$ $\nu_{n}\left(t_{n}\right)$.

The arithmetic/geometric mean inequality and the previous lemma imply that

$$
\nu_{n}\left(s_{n}\right)+\nu_{n}\left(t_{n}\right) \geq 2 \sqrt{c}\left(\frac{6}{64}-\varepsilon\right)^{n / 2} .
$$

Thus

$$
\begin{aligned}
\left.\mid \log \sum_{w \in[s]} \nu_{n}\left(\sigma_{n}(w)\right)\right) \mid & \leq\left|\log \left(\nu_{n}\left(s_{n}\right)+\nu_{n}\left(u_{n}\right)\right)\right| \\
& \leq\left|\log 2 \sqrt{c}(6 / 64-\varepsilon)^{n / 2}\right|
\end{aligned}
$$

As $\varepsilon>0$ was arbitrary, an application of Lemma 6 gives the upper bound on $\overline{\operatorname{dim}}_{l o c} \tilde{\nu}([s])$.

To prove (c) of Theorem 3 we need further technical results.

LEMMA 9. $\operatorname{dim}_{l o c} \tilde{\nu}[3 / 2]=\log (8 / \sqrt{6}) / \log 3$.

Proof. Since $3 / 2=\pi(2,0,2,0, \ldots), 1 / 2=\pi(0,2,0,2, \ldots)$ and $(2,0) \in \Omega^{2}$ is a barrier, it is easy to calculate that $\operatorname{dim}_{l o c} \nu(3 / 2)=\operatorname{dim}_{l o c} \nu(1 / 2)=\log (8 / \sqrt{6}) / \log 3$. The same is true for $\operatorname{dim}_{l o c} \nu(5 / 2)$ by symmetry.

LEMMA 10. For $s \in(1 / 3,2 / 3), \overline{\operatorname{dim}}_{l o c} \nu(s)=\overline{\operatorname{dim}}_{l o c} \nu(s+2)$.

Proof. For $s \in(1 / 3,2 / 3)$ there is a presentation of the form $s=\pi\left(0, x_{2}, x_{3}, \ldots\right)$ where $x_{i} \in \Omega_{0}$ and $\left(x_{2}, \ldots, x_{n}\right)$ is a barrier for all $n$. Moreover, $s+2=\pi\left(3, x_{2}, x_{3}, \ldots\right)$. Thus

$$
\nu_{n}\left(s_{n}\right)=\nu_{1}(\pi(0)) \nu_{n-1}\left(x_{2}, \ldots, x_{n}\right)=\nu_{1}(\pi(3)) \nu_{n-1}\left(x_{2}, \ldots, x_{n}\right)=\nu_{n}\left((s+2)_{n}\right) .
$$

To finish, use (4.1).

We have now established that the two endpoints of the specified interval are attained. To complete the proof we argue in a similar fashion to [7, Thm. 4.6].

LEMMA 11. Choose positive integers $k_{i}, j_{i}$ for $i=1, \ldots, m$ and let $O_{m}=$ $\sum_{i=1}^{m} 2 k_{i}, E_{m}=\sum_{i=1}^{m} 2 j_{i}$ and $N_{m}=O_{m}+E_{m}$. Put

$$
s=\pi\left((2,0)_{k_{1}},(1)_{2 j_{1}},(2,0)_{k_{2}},(1)_{2 j_{2}}, \ldots\right) .
$$


(a) If $N_{m} \leq n \leq N_{m+1}$, then

$$
\nu_{N_{m+1}}\left(s_{N_{m+1}}\right) \leq \nu_{n}\left(s_{n}\right) \leq \nu_{N_{m}}\left(s_{N_{m}}\right)=\left(\frac{\sqrt{6}}{8}\right)^{O_{m}}\left(\frac{3}{8}\right)^{E_{m}} .
$$

(b) If $N_{m-1}+1 \leq n \leq N_{m}+1$, then

$$
\begin{aligned}
\frac{2^{-m}}{8}\left(\frac{\sqrt{6}}{8}\right)^{O_{m}-2 m}\left(\frac{3}{8}\right)^{E_{m}+2 m} & \leq \nu_{n}\left((2-s)_{n}\right) \\
& \leq \frac{1}{8}\left(\frac{\sqrt{6}}{8}\right)^{O_{m-1}-2(m-1)}\left(\frac{3}{8}\right)^{E_{m-1}+2(m-1)}
\end{aligned}
$$

Proof. (a) is straightforward as (2,0), (1) and (2) are barriers.

(b) Note that $2-s=\pi\left(0,(2,0)_{k_{1}-1}, 2,(1)_{2 j_{1}}, 0,(2,0)_{k_{2}-1}, \ldots\right)$. A calculation shows $\nu_{n}\left(2,(1)_{n-2}, 0\right)=\left(3^{n}+3\right) /\left(8^{n} 2\right)$. As both $\left(2,(1)_{2 j_{i}}, 0\right)$ and $(2,0)_{k_{i}}$ are barriers,

$$
\nu_{N_{m}+1}\left((2-s)_{N_{m}+1}\right)=\nu_{1}(\pi(0))\left(\frac{\sqrt{6}}{8}\right)^{O_{m}-2 m} \prod_{i=1}^{m}\left(\frac{3^{2 j_{i}+2}+3}{8^{2 j_{i}+2} 2}\right)
$$

and from this one can deduce the desired inequalities.

Taking limits gives the following corollary.

Corollary 8. Suppose $m / N_{m} \rightarrow 0$ and $N_{m+1} / N_{m} \rightarrow 1$. Then

$$
\lim _{n} \frac{\left|\log \nu_{n}\left(s_{n}\right)\right|}{n \log 3}=\lim _{m} \frac{\left|\log \nu_{N_{m}}\left(s_{N_{m}}\right)\right|}{N_{m} \log 3}=\lim _{n} \frac{\left|\log \nu_{n}\left((2-s)_{n}\right)\right|}{n \log 3} .
$$

If, in addition, $O_{m} / N_{m} \rightarrow \theta \in(0,1)$, then

$$
\begin{aligned}
\lim _{m} \frac{\left|\log \nu_{N_{m}}\left(s_{N_{m}}\right)\right|}{N_{m} \log 3} & =\frac{\theta \log (8 / \sqrt{6})+(1-\theta) \log (8 / 3)}{\log 3} \\
& =\lim _{m} \frac{\left|\log \nu_{N_{m}+1}\left(s_{N_{m}+1}\right)\right|}{\left(N_{m}+1\right) \log 3} .
\end{aligned}
$$

Proof of Theorem 3(c). We have already seen both ends of the interval specified in the statement of the theorem are attained, at 0 and 3/2 (Theorem 3(a) and Lemma $9)$ respectively.

Given any $\alpha$ in the open interval, choose $\theta \in(0,1)$ such that

$$
\alpha=(1-\theta) \frac{\log 8 / 3}{\log 3}+\theta \frac{\log 8 / \sqrt{6}}{\log 3} .
$$

Choose $N$ so large that $N \theta, N(1-\theta) \geq 2$ and define $s$ as in the previous lemma with $k_{i}=\lfloor N i \theta\rfloor$ and $j_{i}=\lfloor N i(1-\theta)\rfloor$. One can check that $s \in(4 / 3,3 / 2)$, thus by Lemma 10 the local dimensions of $\nu$ at $s \pm 1$ coincide. Furthermore, by symmetry, the local dimensions of $\nu$ at $s-1$ and $2-s$ agree. By Lemma $5, \operatorname{dim}_{l o c} \tilde{\nu}([s])$ is the minimum of the local dimensions of $\nu$ at $s, s \pm 1$.

The choices we have made for $k_{i}$ and $j_{i}$ ensure that $m / N_{m} \rightarrow 0, N_{m+1} / N_{m} \rightarrow 1$ and $O_{m} / N_{m} \rightarrow \theta$. Calling upon the previous corollary completes the proof of (c). 
This finishes the proof of Theorem 3 .

REMARK 1. One can also show that the set of local dimensions for the quotient measure of the 2-fold convolution of the uniform Cantor measure on the middle-third Cantor set is the interval $[\log 2 / \log 3, \log 4 / \log 3]$. This is much easier because if $\pi\left(x_{1}, \ldots, x_{n}\right)=\pi\left(z_{1}, \ldots, z_{n}\right)$ for $x_{i}, z_{i} \in\{0,1,2\}$, then $x_{i}=z_{i}$ for all $i$.

We have not been able to determine the multifractal spectrum for the quotient measure of the $k$-fold convolution product when $k \geq 4$ as the calculations are more complicated.

More generally, suppose $m \geq 3$ is an integer and that $\nu_{m}$ denotes the uniform Cantor measure on the central Cantor set with ratio of dissection $1 / m$ at each step. For $m=2 k$ put

$$
A=\left(\begin{array}{c}
m \\
1
\end{array}\right)\left(\begin{array}{c}
m \\
2
\end{array}\right) \cdots\left(\begin{array}{c}
m \\
k-1
\end{array}\right)\left(\begin{array}{c}
m \\
k+2
\end{array}\right) \cdots\left(\begin{array}{c}
m \\
2 k-1
\end{array}\right)\left(\left(\begin{array}{c}
m \\
k
\end{array}\right)+\left(\begin{array}{c}
m \\
k+1
\end{array}\right)\right) .
$$

For $m=2 k+1$, put

$$
A=\left(\begin{array}{c}
m \\
1
\end{array}\right)\left(\begin{array}{c}
m \\
2
\end{array}\right) \cdots\left(\begin{array}{c}
m \\
k-1
\end{array}\right)\left(\begin{array}{c}
m \\
k+2
\end{array}\right) \cdots\left(\begin{array}{c}
m \\
2 k
\end{array}\right)\left(\left(\begin{array}{c}
m \\
k
\end{array}\right)+\left(\begin{array}{c}
m \\
k+1
\end{array}\right)\right) .
$$

The following more general result can be proven by similar, but more complicated arguments. We omit the details.

THEOREM 4. The set of local dimensions for the quotient of the $m$-fold convolution of $\nu_{m}$ is the interval

$$
\left[\frac{-\log \left(\left(\begin{array}{c}
m \\
\lfloor m / 2\rfloor
\end{array}\right) / 2^{m}\right)}{\log m}, \frac{-\log \left(A^{1 /(m-1)} / 2^{m}\right)}{\log m}\right] .
$$

\section{REFERENCES}

[1] C. Cabrelli, K. Hare, and U. Molter, Sums of Cantor sets, Ergodic Theory and Dynamical Systems, 17 (1997), pp. 1299-1313.

[2] K. Falconer, Techniques in fractal geometry, John Wiley and Sons, New York, 1997.

[3] A.-H. FAN, K.-S. LAU, AND S.-M. NGAI, Iterated function systems with overlaps, Asian J. Math., 4 (2000), pp. 243-250.

[4] D.-J. Feng, K.-S. LAU, And X.-Y. WAng, Some exceptional phenomena in multifractal formalism: Part II, Asian J. Math., 9 (2005), pp. 473-488.

[5] K. Hare And T. O'Neil, N-fold sums of Cantor sets, Mathematika, 47 (2000), pp. 243-250.

[6] K. HARE AND S. YAZDANI, Quasi self-similarity and multifractal analysis of Cantor measures, Real Anal. Exch., 27 (2001/2), pp. 287-308.

[7] T.-Y. Hu AND K.-S. LAU, Multifractal structure of convolution of the Cantor measure, Adv. App. Math., 27 (2001), pp. 1-16.

[8] K.-S. LAU AND X.-Y. WANG, Some exceptional phenomena in multifractal formalism: Part I, Asian J. Math, 9 (2005), pp. 275-294.

[9] L. OLsen, Multifractal geometry, Progress in probability, 46 (2000), pp. 3-37.

[10] T. O'NeIL, The multifractal spectrum of quasi-self-similar measures, J. Math. Anal. Appl., 211 (1997), pp. 233-257.

[11] P. Shmerkin, A modified multifractal formalism for a class of self-similar measures with overlap, Asian J. Math, 9 (2005), pp. 323-348.

[12] B. Testud, Phase transitions for the multifractal analysis of self-similar measures, Nonlinearity, 19 (2006), pp. 1201-1217. 
V. P.-W. FONG, K. E. HARE AND D. JOHNSTONE 